\title{
Evaluation comparative des retombées radioactives de l'accident de Tchernobyl et des essais nucléaires atmosphériques
}

\author{
A. DOURY* \\ (Manuscrit reçu le 3 février 1987)
}

\begin{abstract}
RÉSUMÉ
La question de la comparaison des impacts sur l'environnement des retombées radioactives de l'accident de Tchernobyl et des essais nucléaires atmosphériques de 1945 à 1980 mérite une réponse qui présente, entre autres, l'intérêt de fournir des éléments de référence de nature à permettre une appréciation indirecte, facile et correcte de l'impact sanitaire de l'accident par rapport à celui, déjà vécu et perçu comme insignifiant, des essais nucléaires atmosphériques. En fait, quelques réponses à cette question ont déjà été formulées de par le monde, mais il faut bien reconnaître qu'elles ont été variées et souvent inexactes de sorte qu'une mise au point technique objective a paru nécessaire.
\end{abstract}

Si on ne considère que les sources, il est immédiat que les quantités de radioactivité mises en jeu par l'accident de Tchernobyl sont inférieures de un ou plusieurs ordres de grandeur à celles mises en jeu par l'équivalent de $217 \mathrm{Mt}$ de TNT de l'ensemble des essais nucléaires qui ont été effectués de 1945 à 1980 . Comme les différences de contenu initial sont encore renforcées par les différences de rétention à la source, les rapports effectifs résultants pour l'environnement vont, selon les produits, de plusieurs puissances de 10 pour tous les produits ensemble à un peu plus de 100 pour le strontium 90 et à un peu moins de 100 pour le césium 137.

Au niveau des retombées, les résultats sont globalement les mêmes, mais, en raison des comportements différents de produits qui sont émis et dispersés dans des conditions différentes, la répartition est différente. En deçà d'une certaine distance à la source de l'accident, l'impact sanitaire de celui-ci est naturellement plus important que l'impact des essais. Mais, au-delà de cette distance, la retombée de l'accident devient rapidement négligeable devant la retombée globale due à la totalité des essais nucléaires aériens. Cette distance critique est de l'ordre de $1500 \mathrm{~km}$ pour les principaux produits de fission et de quelques centaines de kilomètres pour les actinides.

Un ordre de grandeur de l'impact sanitaire final des différentes retombées peut, en outre, être apprécié simplement si l'on considère que les engagements d'équivalent de dose efficace individuelle provenant de tous les radionucléides libérés dans l'environnement par les essais de 1945 à 1980 correspondent approximativement et en toutes régions à une année moyenne supplémentaire de radioactivité naturelle.

- Commissariat à l'énergie atomique, Institut de protection et de sûreté nucléaire (IPSN), Département d'analyse de sûreté, BP 6, 92265 Fontenay-aux-Roses Cedex, et Service mixte de sécurité radiologique des essais nucléaires, BP 16,91310 Montlhéry. 


\section{ABSTRACT}

The question of the comparison of the environmental impact of the Chernobyl accident to the analogous impact of the atmospheric weapon tests carried out from 1945 to 1980 is worthy answering, and the answer to this question has the advantage of supplying reference data allowing an indirect, easy and good assessment of the health impact of the accident in relation to the impact of the atmospheric tests now perceived as non significative. Several answers have been given throughout the world which were various and often wrong. Consequently, an objective technical statement has seemed necessary.

Considering the sources only, radioactivity amounts from the Chernobyl accident are obviously lower by one or several orders of magnitude than the amounts due to the equivalent of $\mathbf{2 1 7} \mathrm{Mt}$ of TNT from all the nuclear tests carried out from 1945 to 1980 . Since the differences in initial contents are even enhanced by differences in source retention, the resulting effective ratios, for the environment, range, according to the products, from several power of 10 for all the products together, to a little over 100 for strontium 90 and a little under 100 for cesium 137.

For the fallout, the results are wholly the same. However, on account of the different behaviours of products which are released and dispersed in various conditions, their space distribution is different. Within a certain distance from the source, the health impact of the accident is naturally larger than the nuclear test impact. On the contrary, beyond that distance, the accident fallout becomes rapidly negligible vs the global fallout from all the atmospheric nuclear tests. This critical distance is about $1500 \mathrm{~km}$ for the main fission products and some hundred kilometers for actinides.

The magnitude of the final health impact of the various fallouts can moreover be assessed easily by considering that the individual effective dose equivalent commitment resulting from all the radionuclides released into the environment by the 1945-1980 nuclear tests correspond everywhere roughly to an additional mean year of natural radioactivity.

\section{INTRODUCTION}

Après un événement de la nature et de l'importance de la contamination de l'environnement consécutive à la double explosion de la quatrième tranche de la centrale nucléaire de Tchernobyl survenue le 26 avril 1986, il fallait s'attendre à ce que la question de la comparaison des impacts sur l'environnement des retombées radioactives de cet événement et des retombées de même nature, encore en mémoire, des essais nucléaires des trente-cinq années de 1945 à 1980 fût posée.

La question a, en effet, été posée à plusieurs reprises et en différentes occasions, et à notre connaissance les réponses apportées ont été très variées et souvent inexactes.

Mais si les réponses fournies jusqu'à présent sont discutables, il faut reconnaître que la question, quant à elle, est parfaitement justifiée, car plus ou moins consciemment on imagine que si les explications concernant les évaluations directes de l'impact de l'accident sont au moins difficiles à comprendre, quand elles ne sont pas inexactes, la comparaison, si elle est possible, avec un autre impact déja vécu et perçu comme insignifiant peut constituer un élément de référence à la portée de compréhension du plus grand nombre. 


\section{LES SOURCES DE RADIOACTIVITÉ}

Les quantités de radioactivité mises en jeu par les deux catégories d'événements, et éventuellement dispersées dans l'environnement, sont naturellement différentes à la fois en valeurs absolues et en proportions.

\subsection{Les essais}

Pour ce qui concerne les essais, il suffira de se reporter aux publications d'une organisation spécialisée de l'ONU, le Comité scientifique des Nations Unies pour l'étude des effets des rayonnements ionisants (UNSCEAR). De ses publications [13], on peut déduire le tableau I simplifié des énergies et cadences des tirs, groupés selon trois périodes essentielles.

TABLEAU ।

\section{Les tirs aériens}

\begin{tabular}{|c|c|c|c|c|}
\hline Période & Dates & $\begin{array}{c}\text { Durées } \\
\text { (mois) }\end{array}$ & $\begin{array}{c}\text { Energies } \\
\text { (Mt) }\end{array}$ & $\begin{array}{c}\text { Cadences moyennes } \\
\text { (Mt/mois) }\end{array}$ \\
\hline I & juil. 45 - déc. 57 & 150 & 63 & 0,4 \\
II & janv. 58 - oct. 58 & 10 & 29 & 2,9 \\
III & sept. 61 - déc. 62 & 16 & 102 & 6,4 \\
\hline
\end{tabular}

Soit au total $194 \mathrm{Mt}$ à la cadence moyenne de $1 \mathrm{Mt}$ par mois, et $217 \mathrm{Mt}$ si l'on tient compte des tirs français et chinois qui ont suivi jusqu'en 1980. L'unité d'énergie Mt utilisée ici correspond à l'équivalence de 1 mégatonne d'explosif classique TNT, soit:

$$
1 \mathrm{Mt}(\mathrm{TNT})=4,18 \cdot 10^{15} \text { joules }
$$

Comme l'énergie de certains de ces tirs était en partie due au phénomène de fusion nucléaire, et comme nous ne sommes intéressés que par les "produits de fission", il faut encore préciser qu'il ne s'agit tout au long de ce texte que d'énergie de fission, l'énergie réellement développée par certains tirs ayant naturellement été plus ou moins supérieure.

Les rapports de I'UNSCEAR [13] fournissent également les quantités de radioactivité produites par les essais, mais sachant que:

$$
1 \text { fission }=200 \mathrm{MeV}=3,2 \cdot 10^{-11} \text { joules }
$$

et que :

$$
1 \text { Mt }\left(\text { TNT) }=1,308.10^{26}\right. \text { fissions }
$$

il est facile de retrouver ces quantités en utilisant la formule avant décroissance:

$$
A_{i} \text { (becquerels) }=1,308.10^{26} \mathrm{~W} r_{f i} \frac{0,693}{T_{i}}
$$




\section{A. DOURY}

où: $A_{i}=$ quantité produite du radionucléide $\mathrm{i}$ en $\mathrm{Bq}$.

$r_{\mathrm{fi}}=$ rendement de fission (rapide) du produit $\mathrm{i}$.

$\mathrm{T}_{\mathrm{i}}=$ période radioactive du produit de rang $\mathrm{i}$.

$\mathrm{W}=$ énergie de l'essai en Mt d'équivalent TNT.

Pour quelques catégories de produits significatifs susceptibles de se comporter en aérosols, on retrouve ainsi les quantités rassemblées dans le tableau II, qui correspondent à une énergie totale de $217 \mathrm{Mt}$ et à $1 \mathrm{~h}$ de décroissance.

TABLEAU ॥

Produits des tirs aériens

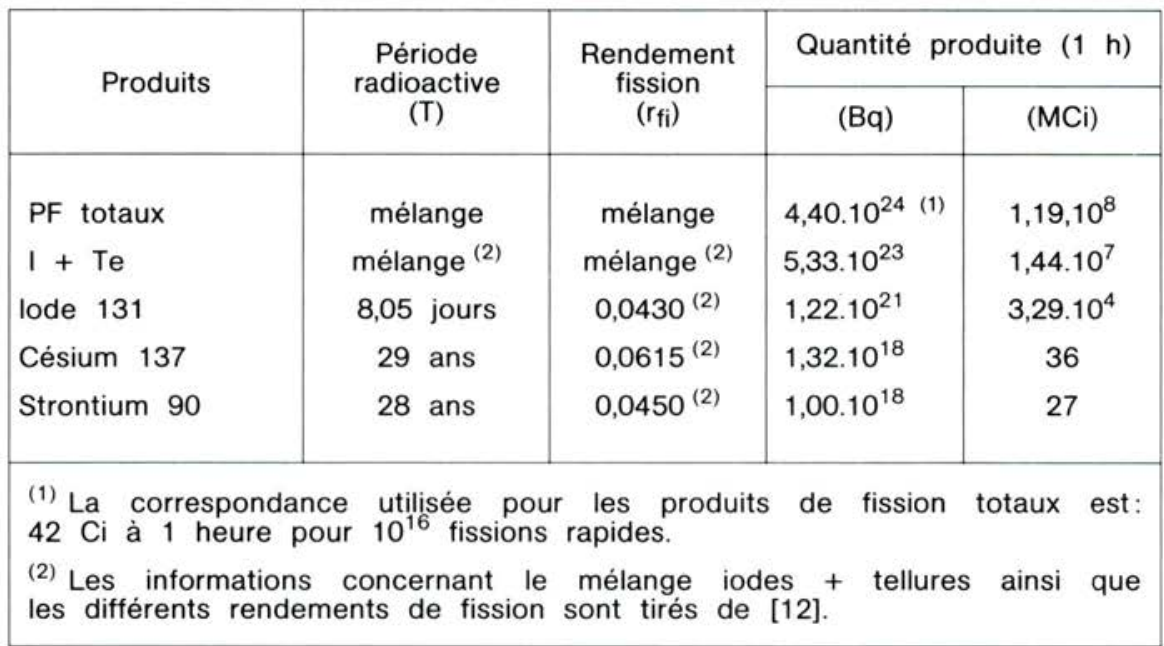

\subsection{L'accident}

Pour l'estimation des quantités de radioactivité, présentes dans le réacteur au moment de l'accident, mais non nécessairement libérées, on adoptera un compromis entre les indications de I'IPSN (CEA) [1], de I'Imperial College de Londres, et des soviétiques eux-mêmes via l'Agence internationale de l'énergie atomique (AIEA). En ne considérant que les produits déjà évoqués et dont la période est assez longue pour qu'on puisse s'en tenir aux valeurs majorantes correspondant à 1 heure de refroidissement, on peut en déduire (tableau III) les quantités exprimées en becquerels à 1 heure, en curies à 1 heure ainsi qu'en équivalence de mégatonnes de TNT d'explosion de fission: 
TABLEAU III

\section{Les produits de l'accident}

\begin{tabular}{|l|c|c|c|c|}
\hline \multirow{2}{*}{ Produits } & \multirow{2}{*}{ Période } & \multicolumn{3}{|c|}{ Quantités $(1 \mathrm{~h})$ présentes dans le réacteur } \\
\cline { 3 - 5 } & & $\mathrm{Bq}$ & $\mathrm{MCi}$ & Eq. Mt TNT \\
\cline { 3 - 5 } & & & & \\
PF totaux & mélange & $4,1.10^{20}$ & $1,1.10^{4}$ & 0,019 \\
$1+\mathrm{Te}$ & mélange & $1,1.10^{19}$ & $3,010^{2}$ & 0,005 \\
lode 131 & 8,05 jours & $2,6.10^{18}$ & $7,1.10^{1}$ & 0,469 \\
Césium 137 & 29 ans & $1,9.10^{17}$ & 5 & 30 \\
Strontium 90 & 28 ans & $1,6.10^{17}$ & 4,4 & 36 \\
\hline
\end{tabular}

\subsection{Comparaisons essais-accident}

En se plaçant uniquement au point de vue des sources potentielles, c'est-à-dire indépendamment des modes de rétention à la source ou de comportement dans l'environnement, on voit donc que les rapports de quantités mises en jeu varient fortement en fonction de la période radioactive des produits considérés. Ce premier résultat n'est pas étonnant, mais on voit aussi que les quantités mises en jeu par l'accident sont toujours inférieures à celles mises en jeu par la totalité des essais aériens, dans la mesure, précisément, où les valeurs de la dernière colonne du tableau III sont inférieures à $217 \mathrm{Mt}$. Le tableau IV, rapport des deux catégories de quantités, est une autre forme de présentation de cette dernière constatation.

TABLEAU IV

Rapports de contenu essais-accident

\begin{tabular}{|c|c|}
\hline Produits & Rapport de contenu essais-accident \\
\hline PFT & 11000 \\
$1+\mathrm{Te}$ & 48000 \\
131 I & 470 \\
${ }^{137} \mathrm{Cs}$ & 7 \\
${ }^{90} \mathrm{Sr}$ & 6 \\
\hline
\end{tabular}

Ce tableau met bien en évidence l'importance de l'effet d'accumulation des produits à vie longue dans un réacteur, alors qu'une explosion libère immédiatement et d'une manière synchrone tous les radioéléments produits. 


\subsection{Commentaires}

Contrairement à ce qui a pu être avancé ici et là [2, 3, 14], il est physiquement impossible que le réacteur soviétique ait libéré dans l'environnement plus et même autant de radioactivité que l'ensemble des essais nucléaires aériens qui ont été répartis sur 35 années de 1945 à 1980. II s'en faut au moins d'un facteur 6 à 50000 selon les produits considérés, et s'il est exact, comme on pouvait le prévoir, que l'écart est assez faible pour les produits à vie longue (à peine un facteur 10 ), il ne faut tout de même pas oublier que, pour ce qui concerne l'accident et seulement dans ce cas, il y a encore lieu de tenir compte des fractions effectivement relâchées, peut-être controversées, mais dont l'existence est indiscutable et qui sont de nature à augmenter encore considérablement les écarts.

\section{LES RETOMBÉES RADIOACTIVES}

Bien que la démonstration que nous nous étions proposée soit déjà acquise en ne considérant que les sources respectives, il n'est pas sans intérêt de vérifier si cette démonstration est confirmée par les retombées tant constatées que calculées. On tentera cet exercice non pas en essayant de remonter avec précision aux quantités relâchées à partir des différentes mesures effectuées dans l'environnement, mais dans l'esprit de la recherche de la cohérence des ordres de grandeur entre les informations disponibles concernant les rejets et celles concernant les sources.

\subsection{Les essais}

Qu'il s'agisse du comportement des polluants (trajectoires et diffusion), de la modélisation de la dispersion et des dépôts, ou des données numériques prévisibles ou observées, il existe une abondante littérature consacrée aux essais nucléaires aériens. II n'est pas nécessaire de revenir ici sur tous les détails de ces différents aspects. Quelques rappels importants concernant les deux premiers sont, cependant, indispensables avant d'aborder le troisième.

Lors d'un essai nucléaire aérien, tous les produits sont immédiatement dispersés dans l'atmosphère et répartis en altitude selon des proportions qui obéissent à des lois bien connues, de sorte que pour suivre l'évolution ultérieure de ces produits il faut considérer un certain nombre de trajectoires simultanées sur un certain nombre de niveaux. L'épaisseur et le nombre de niveaux à considérer, ainsi que la proportion des produits libérés à faire naviguer à chacun de ces niveaux est à déterminer cas par cas, car ces paramètres dépendent à la fois des caractéristiques de l'engin expérimenté et des conditions météorologiques. C'est ainsi que pour un engin de $40 \mathrm{kt}$ le niveau le plus chargé, en atmosphère moyenne, est de l'ordre de $11000 \mathrm{~m}$, sans pour autant que les niveaux les plus bas soient vides de contamination. La détermination des trajectoires est une affaire de météorologistes.

Les trajectoires étant déterminées ou repérées, il reste à apprécier sur chacune d'entre elles la déconcentration des produits en fonction du temps 
sous l'effet de la diffusion naturelle. Cette déconcentration peut être représentée numériquement par un coefficient particulier, dit "coefficient de dilution atmosphérique (CDA)" dont l'expression au maximum, c'est-à-dire sur la trajectoire [9], est la suivante:

$$
\mathrm{CDA}=\frac{1}{(2 \pi)^{3 / 2} \sigma_{\mathrm{x}} \sigma_{\mathrm{y}} \sigma_{\mathrm{z}}}
$$

où, avec les notations habituelles et $x$ sur la trajectoire [8]:

$$
\begin{aligned}
\sigma_{\mathrm{n}}=\sigma_{\mathrm{x}}=\sigma_{\mathrm{y}} & =43,20 \quad \mathrm{t}_{\mathrm{j}} \begin{array}{l}
\text { (écart-type horizontal en } \mathrm{km} \text { jusqu'à } \\
10 \text { jours); }
\end{array} \\
\sigma_{\mathrm{h}}=\sigma_{\mathrm{x}}=\sigma_{\mathrm{y}} & =131,60 \sqrt{\mathrm{t}_{\mathrm{j}}} \begin{array}{l}
\text { (écart-type horizontal en } \mathrm{km} \text { après } \\
10 \text { jours); }
\end{array} \\
\sigma_{\mathrm{z}} & =1,32 \sqrt{\mathrm{t}_{\mathrm{j}}} \begin{array}{l}
\text { (écart-type vertical en } \mathrm{km} \text { ); } \\
\mathrm{t}_{\mathrm{j}}=\text { durée du transfert exprimée en jours. }
\end{array}
\end{aligned}
$$

Près du sol, la valeur de CDA est doublée pour tenir compte d'un effet de réflexion et après 1 jour de transfert, $\sigma_{z}$ est remplacé par $h \sqrt{2 \pi}$ où h est une hauteur de mélange, le plus souvent de l'ordre de $1000 \mathrm{~m}$. Si on s'écarte des trajectoires le CDA local est déduit du CDA maximal par application de lois de décroissance gaussiennes dont les écarts-types sont les paramètres $\sigma_{i}$ déjà utilisés pour calculer le maximum.

Les concentrations étant connues, on procède à l'évaluation des dépôts sur le sol grâce à un coefficient de passage entre les concentrations intégrées sur leur durée et les dépôts, qui est toujours à long terme, quels que soient les processus de dépôt - turbulent ou précipité - de l'ordre de $0,01 \mathrm{~m} \cdot \mathrm{s}^{-1}$.

L'intensité de dépôt sec sur le sol par turbulence est obtenue en multipliant la concentration près du sol par un coefficient $V_{d}$, ou vitesse apparente de dépôt sec, dont la valeur la plus courante est de l'ordre de $0,01 \mathrm{~m} / \mathrm{s}$. L'intensité de dépôt par précipitation est obtenue en multipliant la concentration moyenne dans l'épaisseur soumise à précipitation par un coefficient analogue à $V_{d}$ mais dont l'expression est $f_{p} \wedge h_{p}$, où $\wedge$ est un coefficient de lavage de l'ordre de $10^{-4} \mathrm{~s}^{-1}, h_{p}$ est l'épaisseur soumise à précipitation, le plus souvent de l'ordre de $1000 \mathrm{~m}$, et $\mathrm{f}_{\mathrm{p}}$, fréquence des précipitations, naturellement égale à l'unité pour une précipitation donnée, est de l'ordre de 0,1 en moyenne annuelle en zone tempérée, de sorte qu'à long terme le produit moyen $f_{p} \wedge h_{p}$ est encore de l'ordre de $0,01 \mathrm{~m} \cdot \mathrm{s}^{-1}$.

II reste à rappeler que les gaz rares ne sont pas soumis à dépôt et que tout calcul de retombée au-delà d'une certaine échelle de distance et de temps doit être accompagné d'une correction dite "d'appauvrissement" appliquée aux concentrations. Une telle correction est de la forme:

$$
\mathrm{Ca}=\exp -\frac{\mathrm{V}_{\mathrm{d}} \mathrm{t}}{\mathrm{h}}
$$

Elle cesse d'être applicable au-dessus d'une certaine altitude, de l'ordre de 3000 à $5000 \mathrm{~m}$.

La fonction CDA avec ou sans appauvrissement représentée sur la figure 1 est uniquement valable au sol à partir d'une trajectoire basse et avec une hauteur de mélange de $1000 \mathrm{~m}$. En altitude, notamment pour les essais nucléaires, il y a lieu de rétablir l'écart-type vertical dans l'expression du CDA maximal et d'utiliser cet écart-type pour évaluer, sans correction d'appauvrissement, les concentrations près du sol. 


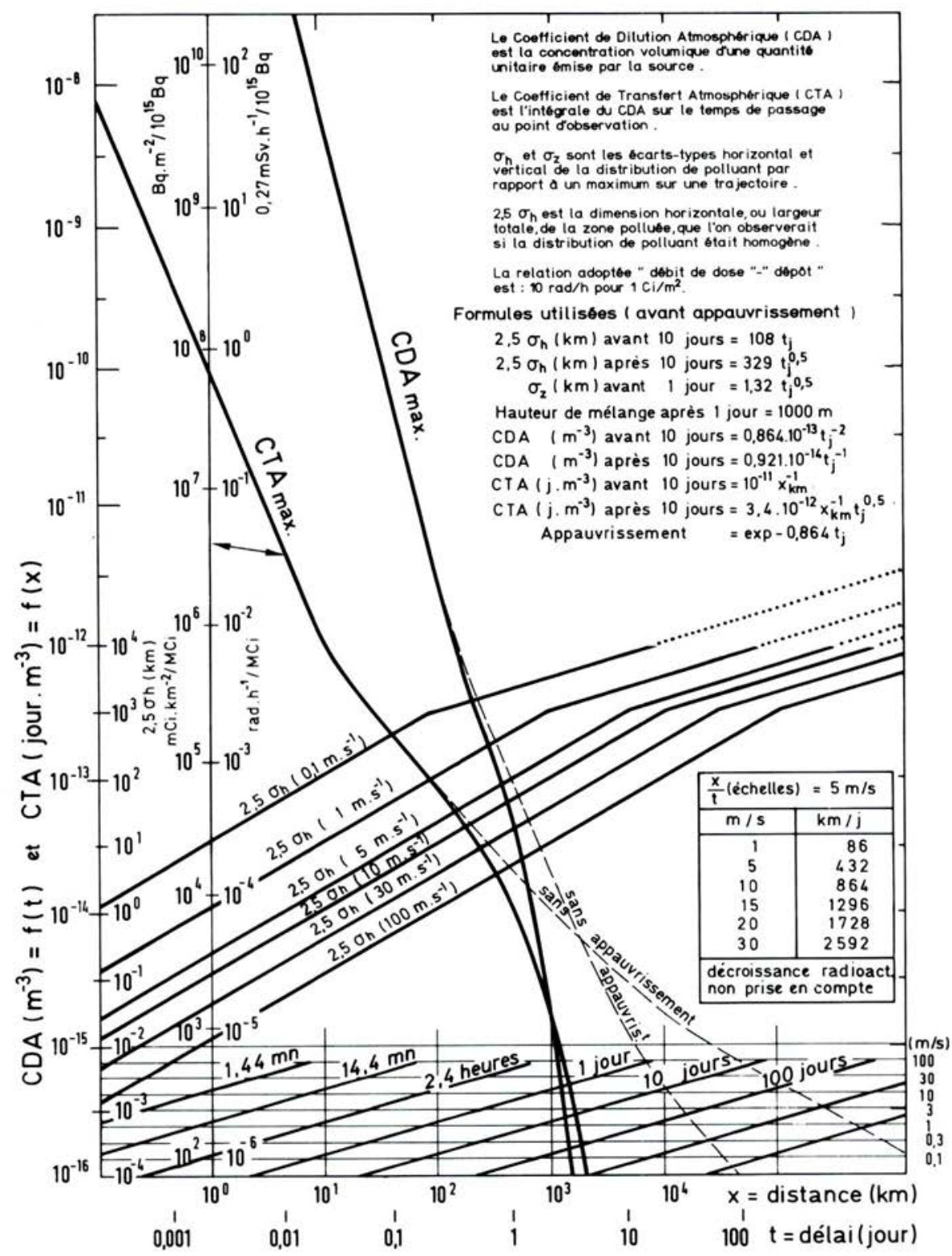

Fig. 1. - Eléments de modélisation simple de la dispersion atmosphérique pour une émission et un transfert dans les basses couches. 
Plus ou moins implicitement, ces démarches très simples se retrouvent à la base de la plupart des modèles.

\section{Données numériques concernant les retombées dues aux essais}

Les données numériques seront traitées selon trois catégories: les produits de fission totaux (PFT) hors gaz rares, l'iode 131, le césium 137 et le strontium 90 , en remarquant que, moyennant une légère transposition, les résultats relatifs au césium 137 seront aussi valables pour le strontium 90, car les périodes de décroissance de ces deux radioéléments (29 et 28 ans) sont pratiquement les mêmes et le rapport de leur production est voisin de l'unité $(\mathrm{Cs} / \mathrm{Sr}=1,33)$.

Les produits de fissions totaux comportent un inconvénient majeur. Ils constituent un mélange disparate de produits aux propriétés différentes et surtout de durées de vie très différentes, de sorte qu'ils se prêtent mal à certains calculs dont l'interprétation, de plus, est malaisée. Mais les moyens requis pour leur mesure en routine sont, par ailleurs, tellement moins compliqués et moins onéreux que ceux exigés par chaque radionucléide pris individuellement, qu'en raison principalement de la disponibilité de leurs mesures en longues séries historiques, au moins depuis 1958, ils sont tout de même de nature à fournir des renseignements très intéressants comme indice de radioactivité artificielle, d'autant que si l'on en connaît l'âge moyen, on a quelques indications sur les proportions de leurs constituants.

D'un très grand nombre de publications provenant d'un grand nombre de laboratoires d'un grand nombre de pays et de plusieurs organisations internationales, on a donc pu extraire l'information fondamentale continue, quelquefois quotidienne et souvent mensuelle, en de nombreuses stations de mesure, que les spécialistes dénomment couramment "indice de radioactivité $\beta$ globale à 5 jours, des poussières atmosphériques". Cette dénomination provient des faits suivants:

- les produits de fission sont des émetteurs $\beta$;

- la radioactivité naturelle des poussières atmosphériques comporte une composante $\beta$ à vie courte qui est toujours restée très supérieure à celle des produits de fission, de sorte que ces derniers, dans un échantillon de mélange, ne peuvent être correctement distingués qu'après un délai de mesure de 5 jours.

A partir de cet indice, exprimé en valeurs moyennes sur certaines périodes, on calcule un dépôt total, dit intégré, sur ces périodes, à l'aide du coefficient de vitesse apparente de dépôt précédemment indiqué, soit avec la correspondante:

$$
1 \text { Bq.j. } \mathrm{m}^{-3} \rightarrow 864 \text { Bq. } \mathrm{m}^{-2}
$$

Des tests de vraisemblance sont ensuite effectués par comparaison avec des mesures directes de dépôt éventuellement disponibles et, en prenant comme référence les trois périodes du tableau I, il est enfin possible de dresser le tableau $\mathrm{V}$ des retombées de produits de fission (hors gaz rares) à 5 jours dues aux essais. 
TABLEAU $V$

Retombées de produits de fission dues aux essais

\begin{tabular}{|c|c|c|c|c|c|c|c|c|}
\hline \multirow{2}{*}{ Période } & \multirow{2}{*}{$\begin{array}{l}\text { Date } \\
\text { des } \\
\text { tirs }\end{array}$} & \multirow{2}{*}{$\begin{array}{c}\begin{array}{c}\text { Durée } \\
\text { des } \\
\text { retomb. }\end{array} \\
\text { (mois) }\end{array}$} & \multicolumn{2}{|c|}{$\begin{array}{l}\text { Concentration } \\
\text { moyenne }\end{array}$} & \multicolumn{2}{|c|}{$\begin{array}{l}\text { Concentration } \\
\text { intégrée }\end{array}$} & \multicolumn{2}{|c|}{ Dépôt } \\
\hline & & & $\left(\mathrm{pCi} \cdot \mathrm{m}^{-3}\right)$ & $\left(\mathrm{Bq} \cdot \mathrm{m}^{-3}\right)$ & $\left(\mathrm{pC}\right.$ i.j. $\left.\mathrm{m}^{-3}\right)$ & (Bq.j. $\mathrm{m}^{-3}$ ) & $\left(\mathrm{mCi} . \mathrm{km}^{-2}\right)$ & $\left(\mathrm{Bq} \cdot \mathrm{m}^{-2}\right)$ \\
\hline$\left(63^{\prime} \mathrm{Mt}\right)$ & $\begin{array}{l}\text { juil. } 45 \\
\text { déc. } 57\end{array}$ & 150 & 0,6 & 0,02 & 2430 & 90 & 2100 & 77800 \\
\hline (29 $\mathrm{Mt})$ & $\begin{array}{c}\text { janv. } 58 \\
\text { oct. } 58 \\
{[4]}\end{array}$ & 18 & 4 & 0,15 & 2160 & 80 & 1870 & 70000 \\
\hline $\begin{array}{c}\text { III } \\
(102 \mathrm{Mt})\end{array}$ & $\begin{array}{l}\text { sept. } 61 \\
\text { déc. } 62 \\
\text { [5] [6] }\end{array}$ & 28 & 5 & 0,19 & 4200 & 156 & 3600 & 134400 \\
\hline $\begin{array}{c}\text { Total } \\
\text { (194 Mt) }\end{array}$ & $\begin{array}{ll}\text { juil. } & 45 \\
\text { déc. } & 62\end{array}$ & 196 & 1,5 & 0,06 & 8790 & 326 & 7600 & 282000 \\
\hline
\end{tabular}

La quantité totale, de 282000 Bq. $\mathrm{m}^{-2}$ pour $194 \mathrm{Mt}$, peut être estimée à $315000 \mathrm{~Bq} \cdot \mathrm{m}^{-2}$ pour $217 \mathrm{Mt}$. Cette valeur correspond à une répartition quasi uniforme sur toute la planète ou au moins sur son hémisphère Nord, car l'essentiel des essais a eu lieu dans l'hémisphère Nord et une grande partie des produits est même passée par la stratosphère avant de retomber sur le sol ou les océans.

Mais, dans ce mélange de produits, on admet généralement, pour différentes raisons physiques et sanitaires, que deux d'entre eux sont assez bien représentatifs des deux plus importantes catégories. II s'agit de l'iode 131, de période 8 jours, représentant les produits à vie relativement courte et le césium 137, de période 29 ans, représentant les produits à vie longue.

Pour l'iode 131, un calcul analogue au précédent est difficilement praticable en raison de sa période trop courte devant la durée des périodes d'essais, qui entraîne notamment qu'il a partiellement le temps de disparaître avant d'atteindre le sol, surtout lorsqu'il est d'abord passé par les hautes altitudes, comme c'est le cas à l'occasion des plus fortes énergies. Mais pour les besoins de la démonstration proposée, on peut se livrer à un exercice intéressant qui consiste à choisir parmi tous les essais l'un d'entre eux qui serait suffisamment isolé pour que sa retombée soit elle-même suffisamment isolée et dont on connaîtrait assez bien à la fois les paramètres de source et des données de retombées. Un tel essai existe. II s'agit du tir chinois de $40 \mathrm{kt}$ du 14 mai 1965, dont on peut estimer [7] que la retombée mesurée à Saclay est due essentiellement à une tranche originelle de $1000 \mathrm{~m}$ d'épaisseur centrée sur une altitude de $11000 \mathrm{~m}$ et contenant approximativement $3,5 \%$ de la quantité totale de produits libérés. Le passage à Saclay du nuage d'aérosols radioactifs dû au tir chinois du 14 mai 1965, précisément 
repéré par son contenu en iode 131, a duré environ 1 mois, du 25 mai au 21 juin [7]. II a provoqué des concentrations dont les valeurs brutes sont indiquées dans le tableau $\mathrm{VI}$.

\section{TABLEAU VI \\ Indice $\beta$ global à vie longue et activités respectives des principaux radioéléments présents sur les filtres atmosphériques de Saclay en mai, juin et juillet 1965, d'après [7].}

\begin{tabular}{|c|c|c|c|c|c|c|c|c|c|c|c|c|}
\hline \multicolumn{2}{|c|}{\begin{tabular}{|c|} 
Dates \\
Mai-Juin-Juillet 1965
\end{tabular}} & \multirow{3}{*}{$\begin{array}{c}\text { Indice } \\
\beta \\
\text { Total } \\
\mathrm{pCi} / \mathrm{m}^{3}\end{array}$} & \multicolumn{10}{|c|}{ Radioéléments } \\
\hline \multirow[t]{2}{*}{$\begin{array}{l}\text { Prélève- } \\
\text { ments }\end{array}$} & \multirow[t]{2}{*}{ Comptage } & & \multicolumn{2}{|c|}{$\begin{array}{c}{ }^{144} \mathrm{Ce}+ \\
{ }^{144} \mathrm{Pr}\end{array}$} & \multicolumn{2}{|c|}{${ }^{141} \mathrm{Ce}$} & \multicolumn{2}{|c|}{131} & \multicolumn{2}{|c|}{${ }^{125} \mathrm{Sb}$} & \multicolumn{2}{|l|}{${ }^{7} \mathrm{Be}$} \\
\hline & & & $\begin{array}{l}\text { Activité } \\
\mathrm{pCi} / \mathrm{m}^{3}\end{array}$ & $\%$ & $\begin{array}{l}\text { Activité } \\
\text { pCi/m } \mathrm{m}^{3}\end{array}$ & $\%$ & \begin{tabular}{|l|} 
Activité \\
$\mathrm{pCi} / \mathrm{m}^{3}$
\end{tabular} & $\%$ & $\begin{array}{l}\text { Activité } \\
\mathrm{pCi} / \mathrm{m}^{3}\end{array}$ & $\%$ & \begin{tabular}{|l|} 
Activité \\
$\mathrm{pCi} / \mathrm{m}^{3}$
\end{tabular} & $\%$ \\
\hline 1 & 2 & 3 & 4 & 5 & 6 & 7 & 8 & 9 & 10 & 11 & 12 & 13 \\
\hline 1 au $7 / 5$ & $10 / 5$ & 0,164 & 0,079 & 48 & 0 & 0 & 0 & 0 & 0,008 & 4,6 & - & - \\
\hline 7 au $13 / 5$ & $16 / 5$ & 0,256 & 0,119 & 46 & 0 & 0 & 0 & 0 & 0,011 & 4,3 & - & - \\
\hline 13 au $19 / 5$ & $22 / 5$ & 0,480 & 0,126 & 26 & 0 & 0 & 0 & 0 & 0,011 & 2,2 & - & - \\
\hline 19 au $25 / 5$ & $28 / 5$ & 0,408 & 0,128 & 31 & 0 & 0 & 0 & 0 & 0,010 & 2,5 & - & - \\
\hline 25 au $1 / 6$ & $3 / 6$ & 0,435 & 0,155 & 36 & 0,009 & 2,1 & 0,028 & 6,5 & 0,006 & 1,3 & - & - \\
\hline 31 au $3 / 6$ & $7 / 6$ & 0,344 & 0,133 & 39 & 0,005 & 1,6 & 0,003 & 0,8 & 0,007 & 2,1 & 0,127 & - \\
\hline 3 au $4 / 6$ & $9 / 6$ & 0,483 & 0,126 & 26 & 0,013 & 2,5 & 0,008 & 1,7 & 0,008 & 1,5 & 0,105 & - \\
\hline 4 au $5 / 6$ & $10 / 6$ & 0,830 & 0,210 & 25 & 0,028 & 3,3 & 0,010 & 1,3 & 0,012 & 1,4 & 0,161 & - \\
\hline 5 au $6 / 6$ & $11 / 6$ & 0,945 & 0,227 & 24 & 0,037 & 3,9 & 0,010 & 1,0 & 0,012 & 1,2 & 0,155 & - \\
\hline 6 au $8 / 6$ & $13 / 6$ & 0,450 & 0,126 & 28 & 0,010 & 2,3 & 0,003 & 0,8 & 0,006 & 1,4 & 0,131 & - \\
\hline 8 au $11 / 6$ & $15 / 6$ & 0,470 & 0,141 & 30 & 0,008 & 1,7 & 0,004 & 0,8 & 0,006 & 1,4 & 0,093 & - \\
\hline 11 au $16 / 6$ & $19 / 6$ & 1,000 & 0,250 & 25 & 0,054 & 5,4 & 0,010 & 1,0 & 0,014 & 1,4 & 0,330 & - \\
\hline 16 au $21 / 6$ & $24 / 6$ & 0,940 & 0,155 & 16,5 & 0,056 & 6,0 & 0,010 & 1,1 & 0,013 & 1,4 & 0,217 & - \\
\hline 21 au $26 / 6$ & $29 / 6$ & 0,860 & 0,164 & 19 & 0,057 & 6,6 & 0 & 0 & 0,009 & 1,1 & 0,250 & - \\
\hline $\begin{array}{|ll|}26 \text { au } & 1 / 7 \\
\end{array}$ & $4 / 7$ & 0,675 & 0,169 & 25 & 0,034 & 5,1 & 0 & 0 & 0,014 & 2,1 & 0,210 & - \\
\hline 1 au $6 / 7$ & $9 / 7$ & 0,595 & 0,174 & 29 & 0,036 & 6,0 & 0 & 0 & 0,010 & 1,7 & 0,223 & - \\
\hline 6 au $11 / 7$ & $14 / 7$ & 0,593 & 0,140 & 24 & 0,041 & 7,0 & 0 & 0 & 0,009 & 1.5 & 0,158 & - \\
\hline 11 au $16 / 7$ & $19 / 7$ & 0,400 & 0,112 & 28 & 0,009 & 2,3 & 0 & 0 & 0,005 & 1,2 & 0,135 & - \\
\hline 16 au $21 / 7$ & $24 / 7$ & 0,290 & 0,087 & 30 & 0,009 & 3,1 & 0 & 0 & 0,004 & 1,5 & 0,139 & - \\
\hline 21 au $26 / 7$ & $29 / 7$ & 0,276 & 0,074 & 27 & 0 & 0 & 0 & 0 & 0,004 & 1,5 & 0,088 & - \\
\hline 26 au $1 / 8$ & $4 / 8$ & 0,221 & 0,076 & 34 & 0 & 0 & 0 & 0 & 0,004 & 1,7 & 0,112 & - \\
\hline
\end{tabular}

Colonnes $5,7,9,11,13$ : pourcentages par rapport à l'activité $\beta$ totale.

Les activités des radioéléments sont ramenées à leur valeur au jour du comptage $\beta$ total (col. 2).

Compte tenu du rendement des filtres, apprécié par le rapport des proportions observées aux proportions théoriques de l'iode dans le mélange, les valeurs réelles nettes pour l'iode 131 sont probablement plus élevées d'un facteur 3 par rapport à celles qui sont indiquées par ce tableau. 
Un calcul de dispersion entre le point zéro et Saclay a, par ailleurs, été effectué selon une méthode dont les principes ont été exposés précédemment.

Le tout est représenté sur la figure 2 qui met en évidence un accord non fortuit portant notamment sur un maximum à partir de 10 jours et réparti sur un mois, de 20 à 30 Bq.m ${ }^{-2}$ d'iode 131 provenant du seul essai de $40 \mathrm{kt}$ du 14 mai 1965. L'existence de ce maximum est due essentiellement à l'altitude de la source. La cohérence de ce résultat avec ceux du tableau $V$ pour les PT totaux (PFT) est difficile à établir, à la fois pour les raisons indiquées plus haut et parce qu'une simple péréquation par rapport aux énergies donnerait une valeur de retombée en iode 131 pour tous les tirs fortement surévaluée du fait de la faiblesse relative des retombées en produits à vie courte provenant des plus hautes altitudes, donc des plus fortes énergies.

Le césium 137 en revanche, tout comme le strontium 90, dont la décroissance sur 35 ans peut être négligée pour ce genre d'évaluation, se prête beaucoup mieux à des calculs de comparaisons avec les produits de fission totaux. Connaissant, en effet, la surface totale de la terre, soit $2,5.10^{14} \mathrm{~m}^{2}$ par hémisphère et en admettant, bien que des transferts interhémisphériques existent à long terme, que les retombées des essais dans l'hémisphère Nord, de loin les plus nombreux, se soient produites de préférence dans le même hémisphère, on aboutit pour $217 \mathrm{Mt}$, soit, selon le tableau II, pour des quantités totales respectives de $10^{18} \mathrm{~Bq}$ de strontium 90 et de $1,32.10^{18} \mathrm{~Bq}$ de césium 137 uniformément réparties sur l'hémisphère Nord, à des dépôts intégrés finals moyens sur cet hémisphère de:

$$
\frac{1,00 \cdot 10^{18}}{2,5.10^{14}}=4000 \text { Bq. } \mathrm{m}^{-2} \text { pour le strontium } 90
$$

et de:

$$
\frac{1,32 \cdot 10^{18}}{2,5 \cdot 10^{14}}=5280 \text { Bq.m }{ }^{-2} \text { pour le césium } 137 .
$$

Cette dernière valeur, à retenir pour la suite, est reportée sur la figure 3. Le dépôt quasi homogène qu'elle implique est justifié par la diversité des points de tirs et par la possibilité, pour un produit à vie longue injecté à diverses altitudes, de retomber intégralement un peu partout après de multiples brassages. La comparaison avec les valeurs du tableau $\mathrm{V}$ est alors immédiate. A partir d'une retombée intégrée en PFT - hors gaz rares - de 282000 Bq. ${ }^{-2}$ pour $194 \mathrm{Mt}$, soit environ 315000 Bq.m ${ }^{-2}$ pour $217 \mathrm{Mt}$, on obtient une proportion pour le césium de:

$$
\frac{5280}{315000}=1,7 \%
$$




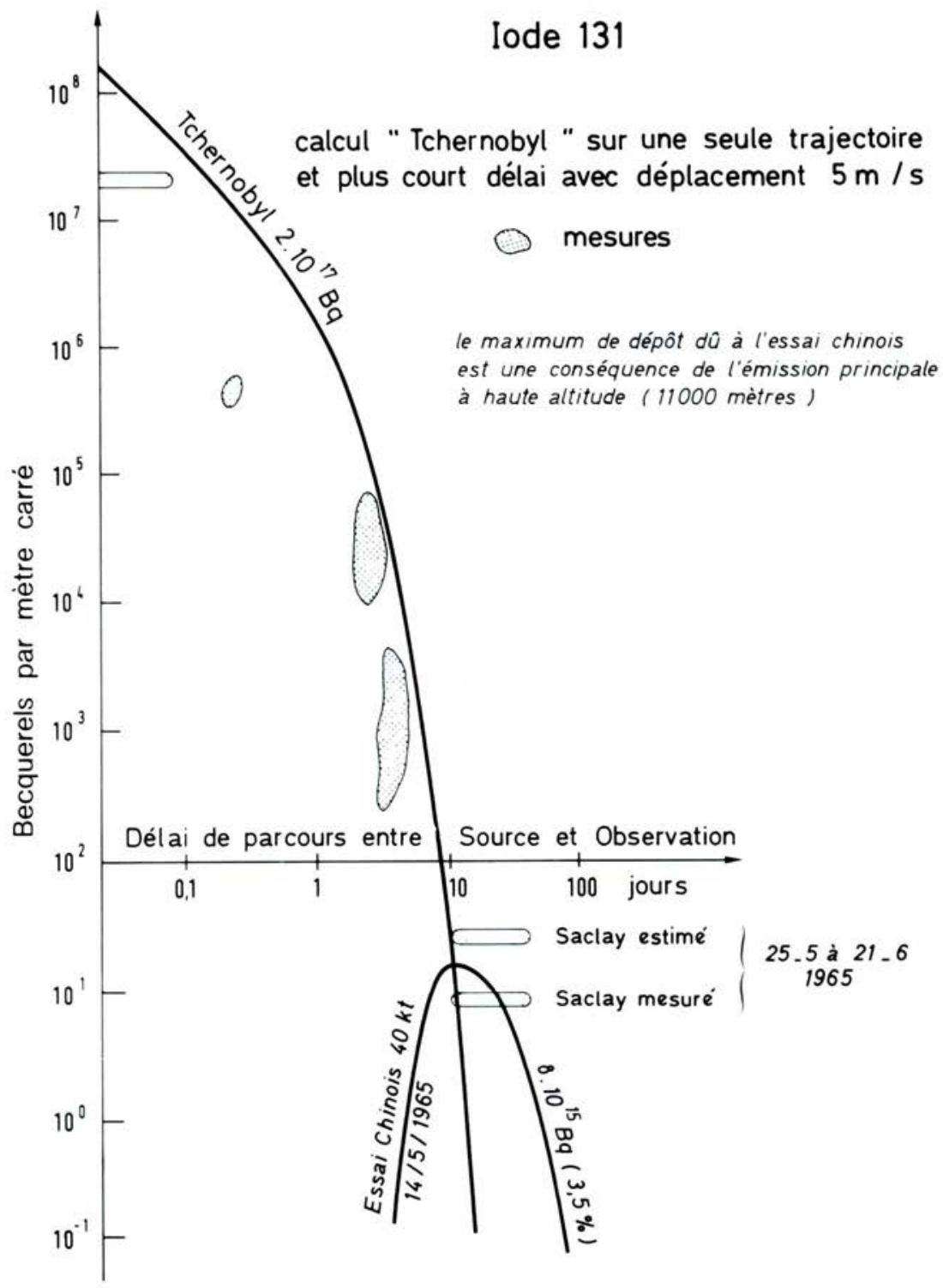

Fig. 2. - Dépôts intégrés comparés d'iode 131 "Tchernobyl” - essais nucléaires. 
Césium 137

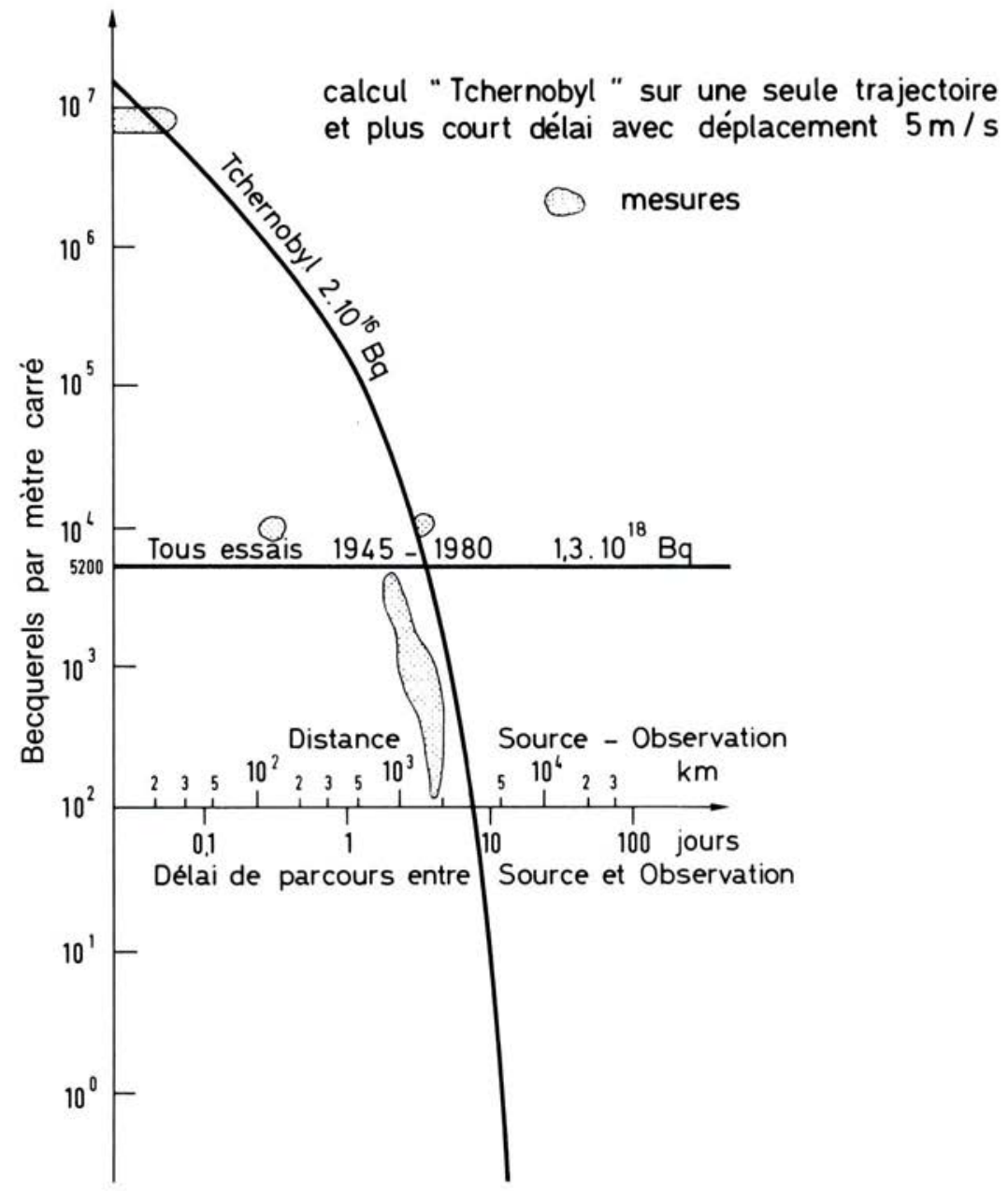

Fig. 3. - Dépôts intégrés comparés de césium 137 "Tchernobyl" essais nucléaires. 
En se limitant aux produits "frais", soit à partir du 25 mai, après 10 jours de transfert, ce résultat est à rapprocher de la production moyenne de $4,3 \%$ que l'on peut déduire du tableau VII relatif à l'essai chinois du 14 mai 1965 [7].

TABLEAU VII

Indice $\beta$ global à vie longue et activités respectives des principaux radioéléments présents sur les filtres atmosphériques de Saclay en mai, juin et juillet 1965 (suite du tableau VI) d'après [7].

\begin{tabular}{|c|c|c|c|c|c|c|c|c|c|c|c|c|}
\hline \multicolumn{2}{|c|}{$\begin{array}{c}\text { Dates } \\
\text { Mai-Juin-Juillet } 1965\end{array}$} & \multirow{3}{*}{\begin{tabular}{|c} 
Indice \\
$\beta$ \\
Total \\
$\mathrm{pCi} / \mathrm{m}^{3}$
\end{tabular}} & \multicolumn{10}{|c|}{ Radioéléments } \\
\hline \multirow[t]{2}{*}{$\begin{array}{l}\text { Prélève- } \\
\text { ments }\end{array}$} & \multirow[t]{2}{*}{ Comptage } & & \multicolumn{2}{|c|}{$\begin{array}{c}{ }^{106} \mathrm{Ru}+ \\
{ }^{106} \mathrm{Rh}\end{array}$} & \multicolumn{2}{|c|}{${ }^{137} \mathrm{Cs}$} & \multicolumn{2}{|c|}{$\begin{array}{c}95 \mathrm{Zr}+ \\
95 \mathrm{Nb}\end{array}$} & \multicolumn{2}{|c|}{${ }^{54} \mathrm{Mn}$} & \multicolumn{2}{|c|}{$\begin{array}{l}{ }^{140} \mathrm{Ba}+ \\
{ }^{140} \mathrm{La}\end{array}$} \\
\hline & & & $\begin{array}{l}\text { Activité } \\
\mathrm{pCi} / \mathrm{m}^{3}\end{array}$ & $\%$ & $\begin{array}{l}\text { Activité } \\
\mathrm{pCi} / \mathrm{m}^{3}\end{array}$ & $\%$ & $\begin{array}{l}\text { Activité } \\
\mathrm{pCi} / \mathrm{m}^{3}\end{array}$ & $\%$ & $\begin{array}{l}\text { Activité } \\
\mathrm{pCi} / \mathrm{m}^{3}\end{array}$ & $\%$ & $\begin{array}{l}\text { Activité } \\
\mathrm{pCi} / \mathrm{m}^{3} \\
\end{array}$ & $\%$ \\
\hline 1 & 2 & 3 & 4 & 5 & 6 & 7 & 8 & 9 & 10 & 11 & 12 & 13 \\
\hline 1 au $7 / 5$ & $10 / 5$ & 0,164 & 0,030 & 18 & 0,014 & 8,6 & 0 & 0 & 0,004 & 2,6 & 0 & 0 \\
\hline 7 au $13 / 5$ & $16 / 5$ & 0,256 & 0,054 & 21 & 0,023 & 8,9 & 0 & 0 & 0,006 & 2,2 & 0 & 0 \\
\hline 13 au $19 / 5$ & $22 / 5$ & 0,480 & 0,068 & 14 & 0,028 & 5,9 & 0 & 0 & 0,008 & 1,7 & 0 & 0 \\
\hline 20 au $25 / 5$ & $28 / 5$ & 0,408 & 0,076 & 18,5 & 0,028 & 6,9 & 0 & 0 & 0,010 & 2,4 & 0 & 0 \\
\hline 25 au $1 / 6$ & $3 / 6$ & 0,435 & 0,074 & 17 & 0,020 & 4,6 & 0,025 & 5,7 & 0,007 & 1,7 & 0,022 & 5 \\
\hline 31 au $3 / 6$ & $7 / 6$ & 0,344 & 0,067 & 19,5 & 0,020 & 6,0 & 0,018 & 5,1 & 0,007 & 2,1 & 0,028 & 8,0 \\
\hline 3 au $4 / 6$ & $9 / 6$ & 0,483 & 0,083 & 17,0 & 0,014 & 2,8 & 0,030 & 6,2 & 0,005 & 1,1 & 0,032 & 6,7 \\
\hline 4 au $5 / 6$ & $10 / 6$ & 0,830 & 0,115 & 14,0 & 0,028 & 3,4 & 0,059 & 7,1 & 0,006 & 0,7 & 0,080 & 9,7 \\
\hline 5 au $6 / 6$ & $11 / 6$ & 0,945 & 0,165 & 17,5 & 0,030 & 3,2 & 0,081 & 8,6 & 0,008 & 0,9 & 0,071 & 7,5 \\
\hline 6 au $8 / 6$ & $13 / 6$ & 0,450 & 0,088 & 19,5 & 0,021 & 4,6 & 0,009 & 1,9 & 0,006 & 1,3 & 0,036 & 8,0 \\
\hline 8 au $11 / 6$ & $15 / 6$ & 0,470 & 0,071 & 15,0 & 0,023 & 5,0 & 0,018 & 3,9 & 0,007 & 1,4 & 0,030 & 6,3 \\
\hline 11 au $16 / 6$ & $19 / 6$ & 1,000 & 0,164 & 16,5 & 0,034 & 3,4 & 0,072 & 7,2 & 0,011 & 1,1 & 0,081 & 8,1 \\
\hline 16 au $21 / 6$ & $24 / 6$ & 0,940 & 0,094 & 10,0 & 0,026 & 2,7 & 0,065 & 6,9 & 0,005 & 0,5 & 0,120 & 12,8 \\
\hline 21 au $26 / 6$ & $29 / 6$ & 0,860 & 0,097 & 11,0 & 0,023 & 2,7 & 0,055 & 6,5 & 0,004 & 0,5 & 0,097 & 11,4 \\
\hline 26 au $1 / 7$ & $4 / 7$ & 0,675 & 0,089 & 13,0 & 0,023 & 3,4 & 0,044 & 6,6 & 0,005 & 0,7 & 0,054 & 8,0 \\
\hline 1 au $6 / 7$ & $9 / 7$ & 0,595 & 0,070 & 11,8 & 0,024 & 4,0 & 0,057 & 9,6 & 0,003 & 0,5 & 0,048 & 8,1 \\
\hline 6 au $11 / 7$ & $14 / 7$ & 0,593 & 0,080 & 13,4 & 0,021 & 3,5 & 0,035 & 5,9 & 0,003 & 0,5 & 0,045 & 7,5 \\
\hline 11 au $16 / 7$ & $19 / 7$ & 0,400 & 0,037 & 9,2 & 0,020 & 5,0 & 0,015 & 3,8 & 0,004 & 0,9 & 0 & 0 \\
\hline 16 au $21 / 7$ & $24 / 7$ & 0,290 & 0,046 & 16,0 & 0,017 & 5,7 & 0,010 & 3,5 & 0,002 & 0,8 & 0 & 0 \\
\hline 21 au $26 / 7$ & $29 / 7$ & 0,276 & 0,045 & 16,4 & 0,018 & 6,4 & 0,005 & 1,8 & 0,003 & 1,0 & 0 & 0 \\
\hline 26 au $1 / 8$ & $4 / 8$ & 0,221 & 0,033 & 14,8 & 0,015 & 6,8 & 0,005 & 2,2 & 0,004 & 1,6 & 0 & 0 \\
\hline
\end{tabular}

Colonnes 5, 7, 9, 11, 13: pourcentages par rapport à l'activité $\beta$ totale.

Les activités des radioéléments sont ramenées à leur valeur au jour du comptage $\beta$ total (col. 2).

Notons ici qu'à un dépôt uniforme de $5200 \mathrm{~Bq} / \mathrm{m}^{2}$ de césium 137 correspond, à 1 mètre du sol, un débit d'iradiation de $1,5.10^{-6} \mathrm{rad} / \mathrm{h}$, soit environ le dixième du débit moyen naturel en terrain non granitique.

VOL. $22 \cdot \mathrm{N}^{\circ} 2$ 


\subsection{L'accident}

Indépendamment des différences à la source indiquées précédemment, il est certain que le comportement ultérieur des produits libérés dans l'environnement par un accident majeur du type de celui de Tchernobyl comporte d'importantes différences par rapport à ceux libérés par les essais nucléaires aériens.

Tout d'abord, lors d'un tel accident, la totalité des produits ne peut jamais être libérée dans l'atmosphère, car si l'on excepte les gaz rares, qui ne représentent que quelques \% des PFT, il existe toujours des phénomènes de rétention dans les structures. En revanche, les altitudes atteintes avant dispersion générale sont beaucoup plus faibles que celles de la plupart des essais de sorte qu'il faut s'attendre, toutes choses égales par ailleurs, en cas d'accident, à des concentrations de pollution plus importantes près des sources et moins importantes aux échelles continentale et mondiale.

Ensuite, et comme pour les essais, il faut distinguer les deux composantes de la dispersion que sont les déplacements en masses sur des trajectoires et la diffusion turbulente. Sur les trajectoires, qui sont comme déjà mentionné, une pure affaire de météorologie, toutes sortes de choses plus ou moins exactes, mais aussi et heureusement plus ou moins déterminantes quant aux suites de cet accident, ont été publiées dans le monde dans un certain désordre, de sorte qu'il est bien difficile, surtout pour un non spécialiste d'y reconnaître les éléments valables et utiles.

Pour ce qui nous concerne ici, il suffira de rappeler que:

- la trajectographie des émissions n'est pas simple;

- les émissions se sont poursuivies pendant 14 jours, et pour l'essentiel du 26 au 30 avril;

- les émissions se sont stabilisées à plusieurs niveaux qui resteront mal connus, probablement entre le sol et $2000 \mathrm{~m}$ pour l'essentiel;

- les processus de précipitation et de remise en suspension ont provoqué de telles hétérogénéités spatiales qu'il est souvent difficile de reconstituer des trajectoires à partir des taches de dépôt observées;

- la diffusion naturelle verticale, de l'ordre de $1000 \mathrm{~m}$ en 24 heures et $2000 \mathrm{~m}$ sur 4 jours, a nécessairement été à l'origine de trajectoires secondaires ou parasites difficiles à prendre en compte dans les calculs;

- les trajectoires calculées par certains services météorologiques par commodité sur des surfaces isobares, c'est-à-dire des trajectoires nécessairement horizontales, peuvent s'écarter notablement, surtout sur de grandes distances, des trajectoires réelles qui comportent des sections à composante verticale;

- l'essentiel des produits libérés entre le 26 et le 30 avril semble avoir été transporté sur 5 trajectoires principales:

- Scandinavie - Finlande: départ 26 avril,

- Pologne - Allemagne - Nord-Est France - lles britanniques: départ 26-27 avril, 
- Tchécoslovaquie - Nord Italie - Sud-Est France: départ 27 avril,

- vers l'Est sans retour: départ 28 avril,

- vers l'Est puis retour sur les Balkans: départ 29-30 avril;

- l'essentiel des produits émis après le 30 avril, soit moins de la moitié du total, n'a pas été dirigé vers l'Europe occidentale.

Devant cette complexité, et compte tenu des marges d'incertitude concernant les émissions, on verra plus loin comment on peut s'en tirer par des raisonnements sur une seule trajectoire théorique.

Pour la diffusion turbulente on dispose de modèles éprouvés $[8,9]$ qui posent beaucoup moins de problèmes que les trajectoires. De plus, parmi ces modèles, on adoptera une version simplifiée [9], tout à fait adaptée à la démonstration poursuivie, et dont le principal élément, très pratique pour le calcul final des dépôts, surtout quand on connaît mal les cadences d'émissions, est l'intégrale - sur la durée du passage sur le récepteur - de la concentration en aérosols due à l'émission d'une quantité unitaire. Cet élément, dénommé "coefficient de transfert atmosphérique (CTA)" est par conséquent le produit du CDA, précédemment défini, par une durée de passage, soit:

$$
(C T A)=(C D A) \cdot \Delta t
$$

Comme le CTA ne dépend pas de la cinétique de l'émission, on peut, avec toujours les mêmes notations, et u étant la vitesse moyenne du vent, se placer dans le cas de l'émission instantanée pour remplacer $\Delta t$ par:

$$
\frac{\Delta x}{\bar{u}}=\frac{\sqrt{2 \pi} \sigma_{x}}{\bar{u}}
$$

pour aboutir, sur la trajectoire, à la relation:

$$
(\mathrm{CTA})_{\max }=\frac{1}{\sqrt{2 \pi} \sigma_{\mathrm{y}} \mathrm{h} \overline{\mathrm{u}}}
$$

d'où, avec les mêmes valeurs d'écarts-types qu'au paragraphe 3.1, une hauteur de mélange moyenne standard de $1000 \mathrm{~m}$, une équivalence moyenne durée-distance $x=u t$, et les transformations convenables pour l'adaptation aux unités de travail choisies, on déduit:

- avant 10 jours avant appauvrissement par dépôt

$$
\begin{array}{ll}
\text { CTA }_{\max } & \begin{array}{l}
\text { (en secondes par mètre cube) } \\
\text { (avec } x \text { exprimé en mètres) }
\end{array} \\
\text { CTA }_{\max } & \text { (en jours par mètre cube) } \\
\text { (avec } x \text { exprimé en kilomètres) } & =10^{-11} \mathrm{x}^{-1}
\end{array}
$$

- après 10 jours avant appauvrissement par dépôt

$$
\begin{aligned}
& \text { CTA }_{\max } \quad \begin{array}{l}
\text { (en secondes par mètre cube) } \\
\text { (avec } x \text { exprimé en mètres) }
\end{array} \\
& \text { CTA }_{\max } \quad \begin{array}{l}
\text { (en jours par mètre cube) } \\
\text { (avec } x \text { exprimé en kilomètres) }
\end{array}=3,4.10^{-6} \mathrm{x}^{-12} \mathrm{x}^{-1} \mathrm{t}^{1 / 2}
\end{aligned}
$$

Les corrections d'appauvrissement et les dépôts sont calculés comme indiqué en 3.1 pour les essais, c'est-à-dire avec la correspondance:

$$
1 \text { Bq.j. } \mathrm{m}^{-3} \rightarrow 864 \text { Bq. } \mathrm{m}^{-2}
$$


Sur la figure 1 où sont rassemblées des courbes de dimensions équivalentes horizontales de nuages de gaz ou aérosols en fonction de la distance ainsi que les courbes de CDA en fonction du temps, on a reporté également les courbes de CTA en fonction de la distance ainsi que des correspondances du CTA en dépôt d'aérosols et en débit d'irradiation due à ce dépôt. II va de soi que les informations portées sur cette figure n'ont que la prétention de fournir rapidement des ordres de grandeur et non celle de remplacer des modèles opérationnels utilisés dans d'autres circonstances. On a toutefois veillé à ce que les deux démarches soient cohérentes.

Découlant plus ou moins directement de ce qui précède, il reste à énoncer trois règles simples qui peuvent rendre certains services:

- l'effet "distance" sur les concentrations intégrées et les dépôts est à peu près inversement proportionnel à la distance,

- l'effet "direction", ou rapport entre valeur sur une seule trajectoire et valeur également répartie dans toutes les directions (rapport entre valeur sur une seule trajectoire et valeur moyenne annuelle), sur les concentrations intégrées et les dépôts est à peu près de l'ordre de 10;

- l'effet "précipitation" sur les dépôts par rapport au dépôt sec est localement et instantanément de l'ordre de 10. On a vu qu'à long terme, et au moins en zones tempérées, il est ramené à 1 .

\section{Données numériques concernant les retombées dues à l'accident}

Comme pour les essais, on distinguera les produits de fission totaux (PFT) hors gaz rares (99\% des PFT dans le réacteur), les iodes et le césium 137, étant entendu que les retombées du strontium 90 sont directement déductibles de celles du césium 137 si l'on considère, pour ces deux radioéléments, la quasi-égalité des périodes de décroissance, les similitudes de comportement au cours de la dispersion dans l'atmosphère et les rapports de production césium / strontium qui sont de l'ordre de l'unité dans le réacteur et, compte tenu d'un taux de rétention du strontium d'environ $96 \%$ à la source, de 3 à 5 à l'extérieur.

Pour les PFT, on dispose de nombreuses mesures provenant, notamment en France, des réseaux permanents du Service central de protection contre les rayonnements ionisants (SCPRI) et du CEA. II ne peut être question d'en faire une présentation exhaustive. II suffira d'indiquer que, selon ces mesures, le passage principal sur la France de l'aérosol de Tchernobyl entre le 30 avril et le 15 mai correspond à une valeur de concentration intégrée remarquablement stable autour de $15 \mathrm{~Bq} . \mathrm{jm}^{-3}$, et qu'après le 15 mai on observe la continuation d'une décroissance régulière jusqu'au retour, courant juillet, aux valeurs de concentrations instantanées quasi permanentes de quelque $10^{-4} \mathrm{~Bq} \cdot \mathrm{m}^{-3}$ antérieures à l'accident et correspondant d'ailleurs plutôt à la chaîne naturelle à vie longue des dérivés du radon qu'à des produits de fission. La figure 4 , provenant du Centre d'études de Montlhéry (SMSR) est une bonne illustration de ces constatations [11].

Compte tenu de ce qui précède, et à partir de cette valeur de 15 Bq.j. $\mathrm{m}^{-3}$, on peut donc estimer le dépôt intégré en France de l'ensemble des produits de fission à environ:

$$
15.864=13000 \text { Bq. } \mathrm{m}^{-2}
$$


RETOMBÉES RADIOACTIVES DE TCHERNOBYL ET DES ESSAIS NUCLÉAIRES ATMOSPHÉRIQUES

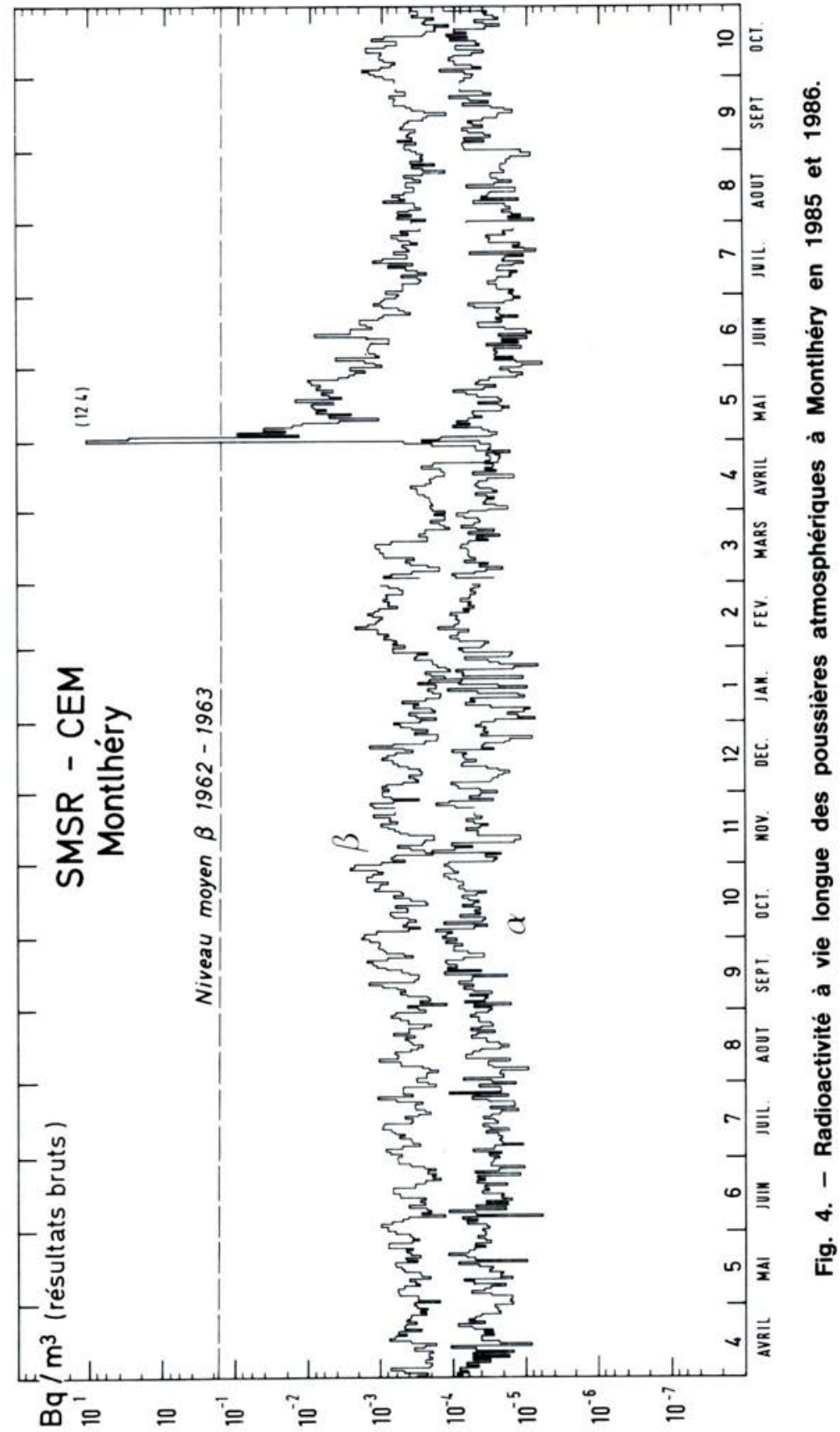

VOL. $22 \cdot \mathrm{N}^{\circ} 2$ 
étant entendu qu'en cas de précipitation sur une concentration maximale d'environ $10 \mathrm{~Bq} \cdot \mathrm{m}^{-3}$ pendant une durée de $6 \mathrm{~h}$, le dépôt aurait pu atteindre localement, et seulement pendant ces 6 heures, une valeur d'environ:

$$
\frac{10 \cdot 864 \cdot 10}{4}=22000 \text { Bq. }{ }^{-2}
$$

supérieure, par conséquent, au total de toute la période. On a là, avec les effets de la météorologie sur les vitesses de dépôt et les remises en suspension, une explication des grandes disparités spatiales constatées.

Si l'on se souvient (tableau V) que l'ensemble des essais nucléaires a entraîné partout d'une manière quasi uniforme, au moins sur l'hémisphère Nord, un dépôt intégré de $315000 \mathrm{~Bq} \cdot \mathrm{m}^{-2}$ de PFT, on voit déjà que pour ces produits, et au moins pour ce qui concerne la France, les retombées de l'accident sont plus de dix fois moindres.

II est intéressant de vérifier maintenant si les éléments de modélisation indiqués sont compatibles avec ces premiers résultats. D'après le tableau III, la quantité de PFT présente dans le réacteur au moment de l'accident est $4.10^{20} \mathrm{~Bq}$. Si pour simplifier on se refère, pour la France, à une distance de $2000 \mathrm{~km}$ parcourus à une vitesse moyenne de $5 \mathrm{~m} \cdot \mathrm{s}^{-1}$, on obtient une durée de transfert de $4.10^{5}$ secondes et un CTA (fig. 1) de $10^{-16}$ jours $/ \mathrm{m}^{3}$. Dans ces conditions, le dépôt théorique maximal pour la quantité totale présente dans le réacteur serait:

$$
4 \cdot 10^{20} \cdot 10^{-16} \cdot 864=35 \cdot 10^{6} \text { Bq.m }{ }^{-2} .
$$

Mais, comme la décroissance d'un mélange de produits de fission provenant d'un réacteur suit à peu près une loi du temps à la puissance - 0,2 [10] et comme on peut estimer que la quantité reçue en France était probablement portée par une trajectoire sur au moins 5 et ne contenait que des produits volatils, soit environ $20 \%$ du total, la valeur précédente doit au moins être réduite par un facteur:

$$
\frac{0,2}{5} \cdot\left(\frac{4.10^{5}}{3600}\right)^{-0,2}=1,6 \cdot 10^{-2}
$$

ce qui donne plus précisément, pour le dépôt théorique maximal en PFT volatils hors gaz rares:

$$
35 \cdot 10^{6} \cdot 1,6 \cdot 10^{-2}=560000 \text { Bq. } \mathrm{m}^{-2}
$$

d'où l'on peut supposer que le taux global de libération des PF volatils dans l'environnement a été de l'ordre de 2,5\% en bon accord avec une émission totale en PF volatils de l'ordre de $2.10^{18} \mathrm{~Bq}$, ou $50 \mathrm{MCi}$, généralement admise, mais plutôt par défaut si l'on tient compte d'un déficit probable des mesures pour les constituants dominants des PF volatils que sont les iodes. Avec les méthodes utilisées et les renseignements disponibles on ne peut pas espérer une meilleure cohérence. 
Les mélanges de PFT présentant toutefois les inconvénients d'interprétation signalés au début, il reste, pour achever la démonstration, à traiter le cas d'un ou deux radionucléides pris séparément. Comme pour les essais et pour les raisons déjà indiquées, on se limitera à l'iode 131 et au césium 137.

Pour ces deux produits, I'IPSN a pu rassembler [1] quelques résultats de mesures exprimés en dépôts intégrés. Le tableau VIII constitue un résumé de mesures européennes auxquelles on peut ajouter des informations plus proches de la source fournies par les soviétiques. L'ensemble est reporté sous forme de différentes plages sur les figures 2 et 3 .

TABLEAU VIII

\section{Quelques résultats de mesures de dépôt d'iode et de césium en Europe après l'accident de Tchernobyl}

\begin{tabular}{|c|c|c|}
\hline \multirow{2}{*}{ Lieu } & \multicolumn{2}{|c|}{ Activité surfacique $\left(\right.$ Bq. $\left.\mathrm{m}^{-2}\right)$} \\
\hline & iode 131 & césium 137 \\
\hline Finlande - Helsinki & $15000-37000$ & $2500-5000$ \\
\hline Suède - Studvisk & $10000-80000$ & $500-2000$ \\
\hline France - Orsay & 600 & 150 \\
\hline G.B. (moyenne) & $500-2700$ & $30-700$ \\
\hline RFA - Münich & $20000-34000(P)$ & 10000 \\
\hline Hollande - Bilthoven & $1800-12000(\mathrm{H})$ & $300-1800(\mathrm{H})$ \\
\hline Danemark - Risø & $600-3000(\mathrm{H})$ & 9 \\
\hline
\end{tabular}

(P) : pluie lors du passage du panache.

$(H)$ : estimé à partir d'une mesure sur l'herbe.

Pour tenter de relier plus précisément ces quelques données aux informations disponibles concernant les émissions, tout en évitant de tomber dans les écueils des incertitudes sur les trajectoires, on propose une évaluation majorante sur une seule trajectoire où l'on suppose, en outre, que le transfert s'effectue sur la plus courte distance depuis l'émission à la vitesse moyenne de $5 \mathrm{~m} / \mathrm{s}$, et on utilise pour la diffusion les mêmes instruments simplifiés que précédemment. On sait que les ordres de grandeur obtenus par cette démarche ont de bonnes chances de rester significatifs ou utilisables car l'hypothèse de plus courte distance est compensée par un vent un peu faible et on ne perd pas de vue que l'hypothèse de trajectoire unique pour la quantité totale émise se traduit par un facteur de majoration de l'ordre de 5 et au plus égal à 10. Tous calculs effectués, en adoptant une hypothèse globale de $10 \%$ d'émission, on obtient finalement deux courbes caractéristiques, l'une pour une quantité libérée de $2.10^{17} \mathrm{~Bq}$ d'iode 131 , l'autre pour une quantité libérée de $2.10^{16} \mathrm{~Bq}$ de césium 137 , qui représentent en Bq. $\mathrm{m}^{-2}$ la quantité totale déposée pendant le passage du nuage d'aérosols à la verticale du maximum de concentration sur la trajectoire. De ce point de vue, il s'agit encore bien d'une valeur maximale qui VOL. $22-\mathrm{N}^{\circ} 2$ 
ne se retrouve pas dans tout l'espace. Pour calculer les valeurs hors trajectoire, il faudrait utiliser les écarts-types prévus par le modèle.

Sur ces mêmes figures 2 et 3 , qui contiennent déjà les informations concernant les retombées d'iode et de césium des essais, on a également reporté ces deux courbes théoriques majorantes ainsi que les plages des résultats de mesures citées relatives à l'accident de manière à faire apparaître clairement:

- les degrés de cohérence, donc de confiance des mesures et des calculs;

- certaines relations fondamentales entre les retombées des essais et celles de l'accident.

Sur la base des évaluations illustrées par les figures 2 et 3, et par rapport à l'estimation globale précédente de 13000 Bq. ${ }^{-2}$ de dépôt de produits volatils à une distance de $2000 \mathrm{~km}$ de la source, il apparaît qu'à cette distance:

- un dépôt théorique d'iode 131 de 8000 Bq. $\mathrm{m}^{-2}$ représente, par excès, $62 \%$ du dépôt total de produits de fission volatils;

- un dépôt théorique de césium 137 de 1600 Bq. $\mathrm{m}^{-2}$ représente, par excès, $12 \%$ du dépôt total de produits de fission volatils;

- le rapport théorique iode/césium égal à 5 découlant de ces calculs est fortement influencé par les hypothèses d'émission et peut être discuté de ce fait.

En considérant que les calculs sont suffisamment confirmés par les mesures, on constate que:

1. il existe, comme on s'y attendait, une distance ou un délai critique en deçà duquel les retombées de l'accident sont plus sévères que celles des essais et au-delà duquel, en revanche, elles deviennent non seulement équivalentes ou moins sévères mais, de plus, rapidement négligeables;

2. fondé sur un seul essai, et a fortiori valable pour l'ensemble des essais, le délai critique pour l'iode 131 est inférieur à 10 jours, mais avec des différences en distances qui proviennent des différences d'altitudes de transfert, donc de vitesses de parcours;

3. la distance critique pour le césium 137 , bon représentant des retombées à vie longue, et a fortiori pour le strontium 90, émis par l'accident dans des proportions moindres que par les essais, est de l'ordre de 1000 à $1500 \mathrm{~km}$, sans influence de vitesse de parcours pour les essais puisque les retombées ultimes de césium 137 et de strontium 90 des essais sont quasi homogènes;

4. les produits à vie courte émis en altitude, notamment par les essais, peuvent disparaître par décroissance radioactive avant de pouvoir toucher le sol ou atteindre de plus grandes distances avant de disparaître.

II faut, de plus, avoir toujours présent à l'esprit que si les retombées dues à l'accident peuvent devenir rapidement très supérieures, de plusieurs ordres de grandeur, à celles des essais lorsqu'on se rapproche de la source, 
elles deviennent en même temps beaucoup plus localisées car il s'agit de valeurs maximales locales sous une trajectoire, tandis que les retombées de produits à vie suffisamment longue dues aux essais sont à peu près uniformément réparties sur au moins un hémisphère tout entier.

\section{CONCLUSIONS}

4.1. A condition de prendre certaines précautions, et surtout de bien connaître les limites de certaines démonstrations, on parvient généralement à retrouver la cohérence qui existe nécessairement entre des émissions de polluants, leur dispersion et leurs retombées dans l'environnement. L'utilisation d'une modélisation de dispersion simple mais suffisante a permis d'éviter les arcanes de la compétition des modèles que cet accident n'a pas manqué de réveiller d'autant plus facilement que les dépôts sont hétérogènes et que les mesures sont toujours discutables et notamment très inégalement réparties dans l'espace.

4.2. Si on ne considère que les sources, il est évident que les quantités mises en jeu par l'accident de Tchernobyl sont inférieures de plusieurs ordres de grandeur à celles mises en jeu par l'ensemble des essais nucléaires effectués de 1945 à 1980 . Comme les différences de contenu initial sont encore renforcées par les différences de rétention à la source, les rapports effectifs résultants pour l'environnement vont, selon les produits, de plusieurs puissances de 10 pour tous les produits ensemble à un peu plus de 100 pour le strontium 90 et à un peu moins de 100 pour le césium 137 .

4.3. Au niveau des retombées, les résultats sont globalement les mêmes, mais, en raison des comportements différents de produits qui sont émis et dispersés dans des conditions différentes, la répartition est différente. En deçà d'une certaine distance à la source, le résultat est naturellement inversé, d'autant plus localement et fortement que l'on se rapproche de cette source. Au-delà, soit au-delà de 1000 à 1500 km pour les seuls produits préoccupants à long terme que sont les produits à vie longue tels que le césium 137 et le strontium 90, la retombée de l'accident devient rapidement négligeable devant la retombée globale due à la totalité des essais nucléaires aériens.

4.4. Des raisonnements de même nature appliqués aux actinides à vie longue conduiraient à des conclusions similaires, avec des distances de renversement généralement encore plus courtes. C'est ainsi que pour le plutonium, dont le rapport de production "essais-accident" est de l'ordre de 100 dans le réacteur et de 2000 à l'extérieur, la distance de renversement n'est plus que de l'ordre de 500 km, avec en outre, des niveaux de radioactivité rapidement non mesurables au-delà de l'environnement proche.

\section{NOTE ADDITIONNELLE SUR L'APPRÉCIATION DES CONSÉQUENCES SANITAIRES}

Bien que strictement hors sujet ici, l'ordre de grandeur de l'impact sanitaire final des différentes retombées évoquées peut être apprécié simplement si l'on considère, en accord avec la publication UNSCEAR 1982 [13, p. 242], que les engagements d'équivalent de dose efficace individuelle 
provenant en toutes régions de tous les radionucléides libérés dans l'environnement par les essais de 1945 à 1980, soit $2100 \mu$ Sv dans l'hémisphère Nord et $700 \mu$ Sv dans l'hémisphère Sud après correction du carbone 14, correspondent approximativement à une année moyenne supplémentaire de radioactivité naturelle.

\section{RÉFÉRENCES}

[1] CEA, IPSN. L'accident de Tchernobyl. Rapport IPSN 2/86, rév. 3, 1986.

[2] DENIS-LEMPEREUR J. Tchernobyl: l'iode s'en va, le césium reste. Sci. Vie, 1986, N. $829,28-35 ; 170$.

[3] DIAMOND S. Long-term Chernobyl fallout comparison to bombs altered. New York Times, Nov. 4, 1986.

[4] DOURY A., FRANÇOIS $\mathrm{H}$. Trois années de mesures quotidiennes de la radioactivité à vie longue au CEN de Saclay (1958-1960). Bull. Inf. Scient. Techn. (BIST), 1961, 56.

[5] DOURY A. Mesures quotidiennes de la radioactivité atmosphérique à longue période sur les principaux sites nucléaires français en 1961 et 1962. Rapport CEA-R-2397, 1963.

[6] DOURY A., CAPUT C. Mesures quotidiennes de la radioactivité atmosphérique à longue période sur les principaux sites nucléaires français en 1963. Rapport CEA-R-2627, 1965.

[7] DOURY $A$, BOURGEON P. Les retombées radioactives en France après la seconde explosion nucléaire chinoise - Processus de transferts atmosphériques. Rapport CEA-R-3020, 1966.

[8] DOURY A. Moyens de calcul opérationnels de la dispersion atmosphérique. Radioprotection, 1982, 17 (2), 83-87.

[9] DOURY A. Une méthode d'approximation instantanée de la dispersion atmosphérique. In: Séminaire sur le transfert à l'homme des radionucléides libérés dans l'environnement par les installations nucléaires, Bruxelles, 17-21 oct. 1983. Luxembourg: Communautés européennes, 1984, 1, 67-77.

[10] LAVIE J.M., DOURY A. Evaluation des risques radioactifs liés au fonctionnement d'une installation nucléaire. Rapport CEA-R-1724, 1960, révisé 1966.

[11] MILLIES-LACROIX J.C., LE LEVIER J. Mesures effectuées au titre de la surveillance de l'environnement consécutivement à l'accident de Tchernobyl. Note technique SMSR-SESE-68, 1986.

[12] SOODAK H., Ed. Reactor handbook, 2. edition. New York: Interscience Publishers, 1962.

[13] UNSCEAR. Ionizing radiation: sources and biological effects. New York: Nations Unies, 1982.

[14] Chernobyl fallout. US To-day, Sept. 24, 1986. 